\title{
RELACÃO DOS PEIXES COLETADOS NOS LIMITES DA PLATAFORMA CONTINENTAL E NAS MONTANHAS SUBMARINAS VITÓRIA, TRINDADE E MARTIN VAZ, DURANTE A CAMPANHA OCEANOGRÁFICA MD-55 BRASIL
}

\author{
José V. Andreata ${ }^{1}$ \\ Bernard Séret ${ }^{2}$
}

\begin{abstract}
LIST OF FISHES COI.IECTFID AT THE CONTINENTAL SHFLF LIMITS VITÓRIA, TRINIDAIE AND MARTIN VAZ SEAMOUNTS DU RING THE MD-55 BRAZII. OCEANOCIRAPIIIC CAMPAIGN. The result of an agreement between Universidade Santa Úrsula, Rio de Janeiro and the Museum of Natural History of Paris, was the oceanographic campaign MD-55 Brasil which took place between May 6th and June 2nd of 1987, aboard the R/V "Marion Dufresne" of the Terres Australes et Antartique Françaises (TAAF). Samples were collected between latitudes $23^{\circ} 36^{\circ} 40^{\prime \prime} \mathrm{S}$ and $18^{\circ} 49^{\circ} \mathrm{S}$. Ichthyofauna sampled was relatively low, and comprised of just one family of Chondrichthyes (three species) and 50 families of Osteichthyes (104 species). Zenion hololepsis (Goode \& Bean. 1895) (Zeniodontidae) is recorded for the first time from the western South Atlantic, as well as is extended the geographic limits for Myrophis frio Jordan \& Davis, 1892 (Ophichthidae) and Prionotus nudigula Ginsburg. 1950 (Triglidae). Even though relatively not very representative, the species collected from the seamount chain Vitoria/Trindade and Martin Vaz suggest being identical to those which occur along the Brazilian continental platform.

KEY WORDS. Fish species list, South Atlantic, continental shelf
\end{abstract}

Um dos primeiros relatos sobre o estudo das espécies de peixes coletados no Atlântico ocidental sul foi de GÜNTHER (1889). A partir deste século a plataforma continental brasileira foi prospetada de norte a sul por vários navios e os resultados foram publicados por diversos autores (Tab. I).

A campanha oceanográfica MD-55 Brasil desenvolveu-se no período de 06 de maio a 02 de junho de 1987, em decorrência de um convênio firmado entre a Universidade Santa Úrsula, Rio de Janeiro e o "Muséum National d'Histoire Naturelle", Paris. O navio utilizado nas pesquisas foi o "Marion Dufresne", pertencente às "Terres Australes Antarctiques Françaises" TAAF.

As coletas de peixes foram realizadas entre as latitudes $23^{\circ} 36^{\circ} 40^{\prime \prime}$ e $18^{\circ} 49^{\circ} \mathrm{S}$, nos limites da plataforma continental e nas montanhas submarinas, Vitória, Trindade e Martin Vaz. A região pesquisada caracteriza-se pela complexidade do relevo, com a presença de montes submarinos e respectivos platôs e bancos. Ao norte da área trabalhada situam-se as zonas de corais; ao sul, situa-se

1) Departamento de Biologia Animal, Universidade Santa Úrsula. Rua Fernando Ferrari 75. 22231-(040 Rio de Janeiro. Rio de Janeiro, Brasil.

2) Ichyologie générale et appliqués. Antenne ORTOM. Muséum National d’Histoire Naturelle. 43. Rue Cuvier. 75231 Paris. France. 
o sistema de ressurgência próximo a Cabo Frio, área cuja fauna é aparentemente mais diversificada.

Este trabalho tem como objetivo produzir uma listagem do material ictiológico obtido na Plataforma Continental e nas montanhas submarinas Vitória, Trindade e Martin Vaz.

Uma relação dos peixes coletados em profundidades maiores do que $200 \mathrm{~m}$ encontram-se em SÉRET \& ANDREATA (1992).

Tabela I. Relação dos navios, referências e anos das principais pesquisas realizadas na plataforma continental brasileira.

\begin{tabular}{|c|c|}
\hline Navio & Referência \\
\hline Annie & RIBEIRO 1903 \\
\hline Oregon & BULLIS JR \& THOMPSON 1959 \\
\hline \multirow{2}{*}{ Tokô-Maru } & RIBEIRO 1961 \\
\hline & NAKAMURA et al. 1963/1969 \\
\hline Doña Maria e Gabriel E. Urias & NOMURA \& FAUSTO FILHO 1966 \\
\hline Ernst Haechel & KARRER 1968 \\
\hline Akaroa, Canops e Almirante Saldanha & ESLINAZI \& LIMA 1968 \\
\hline Almirante Saldanha & OLENCO 1973 \\
\hline Prof. W. Besnard, Emilia & MATSUURA 1971, 1975 \\
\hline \multirow[t]{4}{*}{ Prof. W. Besnard } & SADOWSK। 1971 \\
\hline & MENEZES 1971 \\
\hline & VAZZOLER 1975 \\
\hline & BEVEGNü-LĖ 1978 \\
\hline \multirow[t]{3}{*}{ Riobaldo } & ALVES et al. 1973 \\
\hline & AMARAL 1973 \\
\hline & Carvalho \& Victer 1974 \\
\hline Calypso & Roux 1973, 1979 \\
\hline Malacostraca e Almirante Saldanha & Oliveira $1975,1978,1986$ \\
\hline Cruz del Sur & VAZOLLER et al. 1978 \\
\hline Walther Herwig & MCEACHRAN 1983 \\
\hline Larus & CHAO et al. 1982, 1987 \\
\hline Marion Dufresne & Guille \& Ramos 1988 \\
\hline
\end{tabular}

\section{MATERIAL E MÉTODOS}

As coletas foram realizadas com arrastão de vara "Chalut à Perche" (CP), modelo "TAAF", em operações (esforços) de 15 a 20 minutos. Para os fundos mais duros, foi utilizado o arrastão Blake "Chalut Blake" (CB). Alguns espécimes eventualmente foram capturados pela draga de "Charcot" (DC), usada para coleta do Bentos.

Os espécimes coletados encontram-se na Coleção Ictiológica da Universidade Santa Úrsula, (USU), Rio de Janeiro. Dos lotes com grande número de exemplares, uma pequena amostra foi retirada e registrada na coleção do Muséum National d'Histoire Naturelle (MNHN), Paris. Os poucos exemplares capturados 
de Chondrichthyes foram também registrados no MNHN.

A presente relação inclui, para cada taxon: nome vulgar usual, número da(s) estação(ões) de coleta, instrumento(s) de captura, coordenadas geográficas e distribuição batimétrica. A ordenação das famílias segue NELSON (1984).

\section{RESULTADOS}

Foram identificadas 107 espécies de peixes pertencentes a 51 famílias e 91 gêneros, de acordo com a seguinte listagem:

\section{CHONDRICHTHYES}

\section{Rajidae}

Psammobatis lentiginosa McEachran, 1983

Estação 60 CB $101\left(22^{\circ} 58^{\circ} \mathrm{S}, 42^{\circ} 06^{\prime} \mathrm{W}\right), 50 \mathrm{~m}$.

Raja (Atlantoraja) castelnaui Ribeiro, 1907 - raia-chita

Estação 60 CB $101\left(22^{\circ} 58^{\circ} \mathrm{S}, 42^{\circ} 06^{\prime} \mathrm{W}\right), 50 \mathrm{~m}$.

Raja (Rioraja) agassizi (Müller \& Henle, 1841) - raia-santa

Estação $60 \mathrm{CB} 101\left(22^{\circ} 58^{\prime} \mathrm{S}, 42^{\circ} 06^{\circ} \mathrm{W}\right), 50 \mathrm{~m}$.

\section{OSTEICHTHYES}

\section{Chlopsidae}

Chlopsis bicolor Rafinesque, 1810

Estação 35 DC 59 (22ํํ․

\section{Muraenidae}

Channomuraena vittata (Richardson, 1844) - moréia

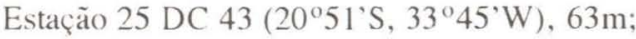

Gymnotorax funebris Ranzani, 1839

Estação 14 DC 27 (203' S, 36 $\left.42^{\circ} \mathrm{W}\right), 81 \mathrm{~m}$; Estação 20 DC 34 (20⒉

$\left.68^{\prime \prime S}, 35^{\circ} 54^{\prime} 45^{\prime \prime W}\right), 54 \mathrm{~m}$; Estação $23 \mathrm{DC}^{\prime} 4 \mathrm{I}^{\prime}\left(20^{\circ} 35^{\prime} 50^{\prime \prime} \mathrm{S}, 34^{\circ} 42^{\prime} 93^{\prime \prime} \mathrm{W}\right.$ ),

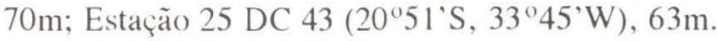

Gymnothorax moringa (Cuvier, 1829) - moréia

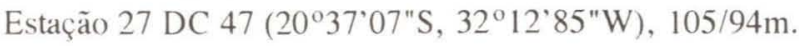

Gymnothorax ocellatus Agassiz, 1831

Estação 6 DC $15\left(21^{\circ} 37^{\circ} 07^{\prime \prime S}, 40^{\circ} 18^{\circ} 01^{\prime \prime W}\right), 37 \mathrm{~m}$; Estação 13 DC 26

$\left(20^{\circ} 21^{\prime} 11^{\prime \prime S}, 36^{\circ} 59^{\circ} 07^{\prime \prime W}\right), 97,5 \mathrm{~m}$; Estação 24 DC 42 (20⒌ $54^{\prime} 67^{\prime \prime S}$,

$\left.34^{\circ} 01^{\prime} 35^{\prime \prime} \mathrm{W}\right), 60 \mathrm{~m}$; Estação 27 DC 47 (20⒋ 'S, 32 $\left.12^{\prime} 85^{\prime \prime W}\right), 94 / 105 \mathrm{~m}$.

Uropterygius acutus (Parr, 1930)

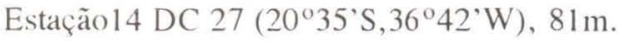

Uropterygius wheeleri Blache, 1967

Estação 32 DC 52 (21 $\left.37^{\circ} 07^{\prime \prime} \mathrm{S}, 48^{\circ} 18^{\circ} 01^{\prime \prime} \mathrm{W}\right), 37 \mathrm{~m}$. 


\section{Ophichthidae}

Myrophis firio Jordan \& Davis, 1892

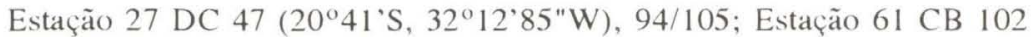
(2307's, $\left.42^{\circ} 3^{\prime} \mathrm{W}\right), 100 \mathrm{~m}$.

Ophichthus gomesi (Castelnau, 1855)

Estação 21 DC 35 (2042'S, 3522'W), 82m; Estação 27 DC 47 (2041'S, $\left.32^{\circ} 12^{\prime} 85^{\prime \prime W}\right), 94 / 105 \mathrm{~mm}$; Estação $60 \mathrm{CB} 100$ (22 ${ }^{\circ} 59^{\circ} 04^{\prime \prime} \mathrm{S}, 42^{\circ} 06^{\circ}$ $\left.55^{\prime \prime W}\right), 45 / 33 \mathrm{~m}$; Estação $60 \mathrm{CB} 101$ (22 $\left.{ }^{\circ} 58^{\prime} \mathrm{S}, 42^{\circ} 06^{\circ} \mathrm{W}\right), 50 \mathrm{~m}$; Estação 61 CB $102\left(23^{\circ} 07^{\prime} \mathrm{S}, 42^{\circ} 03^{\prime} \mathrm{W}\right), 100 \mathrm{~m}$.

Ophichthus parilus (Richardson, 1844)

Estação $60 \mathrm{CB} 100\left(22^{\circ} 59^{\prime} 04^{\prime \prime} \mathrm{S}, 42^{\circ} 06^{\prime} 55^{\prime \prime} \mathrm{W}\right), 45 / 33 \mathrm{~m}$.

\section{Congridae}

Ariosoma opisthophtalma (Ranzani, 1838)

Estação 14 DC 27 (22 $58^{\circ}$ 'S, 42 $\left.{ }^{\circ} 06^{\prime \prime W}\right), 50 \mathrm{~m}$; Estação 60 CB 100 (22 ${ }^{\circ} 59^{\prime}$

$\left.04^{\prime \prime S}, 42^{\circ} 06^{\prime} 5^{\prime \prime W}\right), 45 / 33 \mathrm{~m}$, Estação 60 CB 101 (22 $\left.{ }^{\circ} 58^{\prime} \mathrm{S}, 42^{\circ} 06 \mathrm{~W}\right), 50 \mathrm{~m}$.

Gnathophis sp. aff. mystax (Delaroche, 1809)

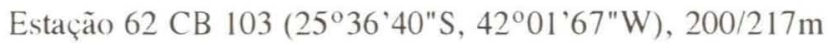

\section{Muraenesocidae}

Cynoponticus savamna (Bancroft, 1831)

Estação 14 DC 27 (22ํำ'S, $\left.42^{\circ} 06^{\prime} \mathrm{W}\right), 50 \mathrm{~m}$; Estação 24 DC 42 (20 $54^{\prime}$ $\left.67^{\prime \prime S}, 34^{\circ} 01^{\prime} 35^{\prime \prime W}\right)$; Estação 25 DC 43 (205 I'S, 334' 'W), 63m.

\section{Clupeidae}

Chirocentrodon bleckerianus (Poey, 1867)

Estação 52 CB 90 (19³3’s, 39³4'W), 34m.

Odontognathus mucronatus Lacépède, 1800

Estação 52 CP 90 (19³3’s, 39³4'W), 34m.

Pellona harroweri (Fowler, 1917)

Estação 51 CP 88 (19³5’s, 3940’W), 20m.

Odontognathus mucronatus Lacépède, 1800

Estação 52 CP 90 (19³3'S, 39³4'W), 34m.

Pellona harroweri (Fowler, 1917)

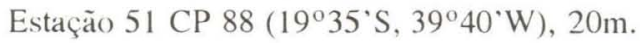

\section{Ariidae}

Bagre marimus (Mitchill, 1814) - bagre-bandeira

Estação 51 CP 88 (19\%35’s, 3940’W), 20m. 


\section{Argentinidae}

Glossanodon pygmaeus Cohen, 1958

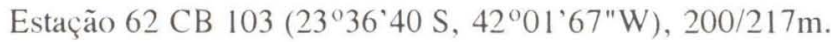

\section{Chlorphthalmidae}

Chlorophthalmus agassizi Bonaparte, 1840

Estação 62 CB 103 (2336’40’S, 4201'67"W). 200/217m.

\section{Synodontidae}

Saurida brasiliensis Norman, 1935 - peixe-lagarto

Estação 52 CB 90 (19033'S, 39³4'W), 34m; Estação 60 CB 101 (22 ${ }^{\circ} 58^{\circ} \mathrm{S}$,

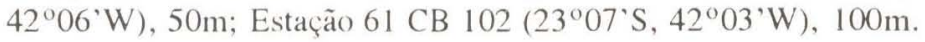

Synodus foetens (Linnaeus, 1766) - peixe-lagarto

Estação 13 DC 26 (20⒈'S, 36 $\left.{ }^{\circ} 59^{\circ} 07^{\circ} \mathrm{W}\right), 97,5 \mathrm{~m}$.

Synodus intermedius: (Spix, 1829)

Estação 13 DC 26 (2011"S, 3659 $\left.07^{\circ} \mathrm{W}\right), 97,5 \mathrm{~cm}$; Estação 27 DC 47 $\left(20^{\circ} 41^{\prime} \mathrm{S}, 32^{\circ} 12^{\circ} 85^{\prime \prime} \mathrm{W}\right), 94 / 105 \mathrm{~m}$; Estação $52 \mathrm{CB} 90$ (19 $33^{\circ} \mathrm{S}$, $\left.39^{\circ} 34^{\prime} \mathrm{W}\right), 34 \mathrm{~m}$; Estação $60 \mathrm{CB} 100\left(22^{\circ} 59^{\circ} 04^{\prime \prime} \mathrm{S}, 42^{\circ} 06^{\circ} 55^{\prime \prime} \mathrm{W}\right), 45 / 33 \mathrm{~m}$.

Trachinocephalus myops (Forster, 1801) - peixe lagarto

Estação 52 CB $90\left(19^{\circ} 33^{\circ} \mathrm{S}, 39^{\circ} 34^{\prime} \mathrm{W}\right), 34 \mathrm{~m}$.

\section{Bregmacerotidae}

Bregmaceros atlanticus Goode \& Bean, 1886

Estação $60 \mathrm{CB} 101\left(23^{\circ} 07^{\prime} \mathrm{S}, 42^{\circ} 03^{\prime \prime W}\right), 100 \mathrm{~m}$; Estação $61 \mathrm{CB} 102$ $\left(23^{\circ} 07^{\circ} \mathrm{S}, 42^{\circ} 03^{\circ} \mathrm{W}\right), 100 \mathrm{~m}$; Estação $62 \mathrm{CB} 103\left(23^{\circ} 36^{\circ} 40^{\prime \prime} \mathrm{S}, 42^{\circ} 01^{\prime}\right)$ $133-176 \mathrm{~mm}$.

\section{Merlucciidae}

Merluccius hubbsi Marini, 1933 - merluza

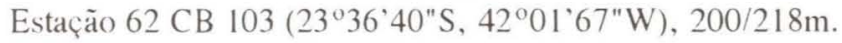

\section{Ophidiidae}

Genypterus blacodes (Schneider, 1801) - Congrio rosado

Estação $61 \mathrm{CB} 102\left(23^{\circ} 07^{\circ} \mathrm{S}, 42^{\circ} 03^{\circ} \mathrm{W}\right), 100 \mathrm{~m}$.

Ophidion holbrooki (Putnam, 1874)

Estação $60 \mathrm{CB} 100$ (22 $\left.{ }^{\circ} 59^{\circ} 04^{\prime \prime} \mathrm{S}, 4^{\circ} 00^{\prime} 55^{\prime \prime W}\right), 45 / 33 \mathrm{~m}$; Estação $60 \mathrm{CB}$

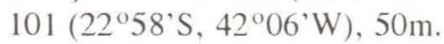

Raneya fluminensis (Ribeiro, 1903)

Estação 52 CB $90\left(19^{\circ} 33^{\prime} \mathrm{S}, 39^{\circ} 34^{\circ} \mathrm{W}\right), 34 \mathrm{~m}$; Estação $60 \mathrm{CB} 100\left(22^{\circ} 59^{\prime}\right.$

$\left.04^{\prime \prime S}, 42^{\circ} 06^{\circ} 55^{\prime \prime W}\right), 45 / 33 \mathrm{~m}$; Estação $60 \mathrm{CB} 101\left(23^{\circ} 07^{\circ} \mathrm{S}, 42^{\circ} 03^{\prime} \mathrm{W}\right)$,

$100 \mathrm{~m}$; Estação $61 \mathrm{CB} 102\left(23^{\circ} 07^{\circ} \mathrm{S}, 42^{\circ} 03^{\circ} \mathrm{W}\right)$. 


\section{Batrachoididae}

Porichthys porosissimus (Valenciennes, 1837) - mamanguá-liso

Estação 13 DC 26 (20021'11"S, 36 $\left.59^{\circ} 07^{\prime \prime W}\right), 97,5 \mathrm{~m}$; Estação 52 CB 90 $\left(19^{\circ} 33^{\circ} \mathrm{S}, 39^{\circ} 34^{\prime \prime W}\right), 34 \mathrm{~m}$; Estação $60 \mathrm{CB} 100\left(22^{\circ} 59^{\circ} 04^{\prime \prime} \mathrm{S}, 42^{\circ} 06^{\circ}\right.$ $\left.55^{\prime \prime} \mathrm{W}\right), 45 / 33 \mathrm{~m}$; Estação $60 \mathrm{CB} 101\left(22^{\circ} 58^{\circ} \mathrm{S}, 42^{\circ} 06^{\circ} \mathrm{W}\right), 50 \mathrm{~m}$; Estação $61 \mathrm{CB} 102,\left(23^{\circ} 07^{\circ} \mathrm{S}, 42^{\circ} 03^{\circ} \mathrm{W}\right), 100 \mathrm{~m}$; Estação $61 \mathrm{CB}^{\circ} 102,\left(23^{\circ} 07^{\prime} \mathrm{S}\right.$,

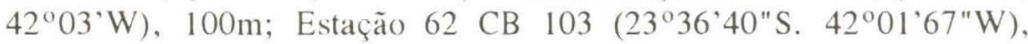
$200 / 217 \mathrm{~m}$.

\section{Ogcocephalidae}

Ogcocephalus vespertilio (Linnaeus, 1758) - peixe morcego

Estação $60 \mathrm{CB} 101\left(22^{\circ} 58^{\circ} \mathrm{S}, 42^{\circ} 06^{\circ} \mathrm{W}\right), 50 \mathrm{~m}$.

\section{Holocentridae}

Sargocentron bullisi (Woods, 1955)

Estação 48 DC 83 (18 $\left.49^{\circ} \mathrm{S}, 37^{\circ} 57^{\circ} \mathrm{W}\right), 60 \mathrm{~m}$.

\section{Zeniontidae}

Zenion hololepis (Goode \& Bean, 1895)

Estação 9 DC $22\left(20^{\circ} 32^{\circ} 09\right.$ S, 38 $\left.30^{\circ} 49^{\prime \prime} \mathrm{W}\right) 52 \mathrm{~m}$.

Obs.: primeira ocorrência para o Brasil.

\section{Caproidae}

Antigonia capros Lowe, 1843

Estação $61 \mathrm{CB} 102\left(23^{\circ} 07^{\prime} \mathrm{S}, 4206^{\circ} \mathrm{W}\right), 100 \mathrm{~m}$; Estação $62 \mathrm{CB} 103$.

Syngnathidae

Hippocampus reidi Ginsburg, 1933 - cavalo-marinho

Estação $11 \mathrm{CP} 25\left(20^{\circ} 35^{\prime} 80^{\prime \prime} \mathrm{S}, 37^{\circ} 27^{\prime} 40^{\prime \prime} \mathrm{W}\right), 65 \mathrm{~m}$; Estação $60 \mathrm{CB} 100$ $\left(22^{\circ} 59^{\circ} 04^{\prime \prime} \mathrm{S}, 42^{\circ} 06^{\circ} 55^{\prime \prime} \mathrm{W}\right) 45 / 33 \mathrm{~m}$; Estação $60 \mathrm{CB} 101{ }^{\circ}\left(22^{\circ} 58^{\circ} \mathrm{S}, 42^{\circ}\right.$ $\left.06^{\prime} \mathrm{W}\right), 50 \mathrm{~m}$; Estação $61 \mathrm{CB} 102\left(23^{\circ} 07^{\prime} \mathrm{S}, 42^{\circ} 03^{\prime} \mathrm{W}\right) 100 \mathrm{~m}$.

\section{Dactylopteridae}

Dactylopterus volitans (Linnaeus, 1758) - coió

Estação 60 CB $100\left(22^{\circ} 59^{\circ} 04^{\prime \prime} \mathrm{S}, 42^{\circ} 06^{\prime} 55^{\prime \prime} \mathrm{W}\right), 45 / 33 \mathrm{~m}$.

\section{Scorpaenidae}

Pontinus rathbuni Goode \& Bean, 1896

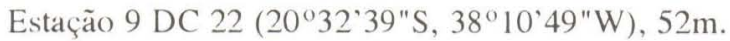

Scorpaena brasiliensis Cuvier, 1829

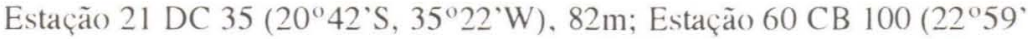


$\left.04^{\prime \prime S}, 42^{\circ} 06^{\prime} 55^{\prime \prime} \mathrm{W}\right), 45 / 33 \mathrm{~m}$.

Scorpaena dispar Longley \& Hildebrand, 1940

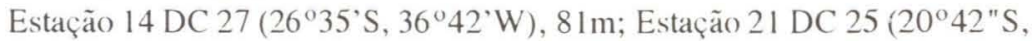

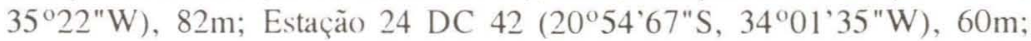
Estação 27 DC 47 (20041'S, 32 $\left.{ }^{\circ} 12^{\prime} 85^{\prime \prime} \mathrm{W}\right), 94 / 105 \mathrm{~m}$; Estação 47 DC 82 (18 $\left.56^{\circ} 35^{\prime \prime S}, 37^{\circ} 52^{\prime} 07^{\prime \prime W}\right), 105 \mathrm{~m}$.

Scorpaena plumieri Bloch, 1789 - mangangá

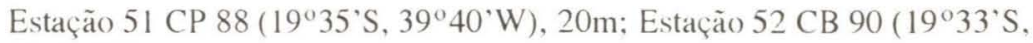
$\left.39^{\circ} 34^{\prime} \mathrm{W}\right), 34 \mathrm{~m}$.

\section{Triglidae}

Bellator brachychir (Regan, 1914)

Estação 61 CB 102 (19³4'S, 39³2’W), 34/48m; Estação 62 CB 103 (19³4'S, 39³2'W), 200/217m.

Prionotus nudigula Ginsburg, 1950 - cabrinha

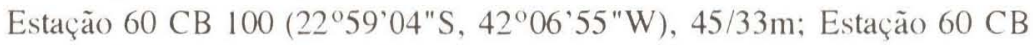
101 (22 $\left.{ }^{\circ} 58^{\prime} \mathrm{S}, 42^{\circ} 06^{\prime} \mathrm{W}\right), 50 \mathrm{~m}$.

Prionotus punctatus (Bloch, 1797) - cabrinha

Estação 52 CP 89 (19³4'S, 39³2'W), 34/48m; Estação 52 CB 90

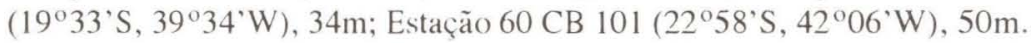

\section{Apogonidae}

Apogon pseudomaculatus Longley, 1932

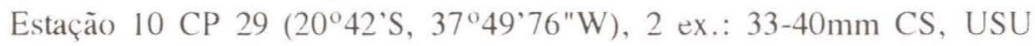
01295.

\section{Acropomatidae}

Synagrops spinosus Schultz, 1940

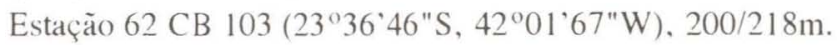

\section{Serranidae}

Dules auriga Cuvier, 1829 - Mariquita

Estação 60 CB 101 (22058’S, 4206’W), 50m.

Serranus phobe Poey, 1851

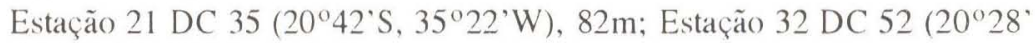
$\left.53^{\prime \prime} \mathrm{S}, 28^{\circ} 51^{\prime} 65^{\prime \prime} \mathrm{W}\right), 120 \mathrm{~m}$.

\section{Carangidae}

Caranx crysos (Mychill, 1815) - Carapau

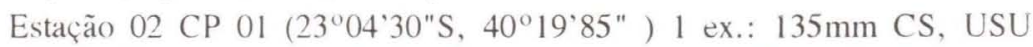
01013 ; Estação 60 CB 100 (220 $\left.19^{\prime} 04^{\prime \prime S}, 42^{\circ} 06^{\prime} 55^{\prime \prime W}\right), 45-33$, I ex.: 
$200 \mathrm{~mm}$ CS, USU 01010.

Trachurus lathami Nichols, 1920 - Xixarro

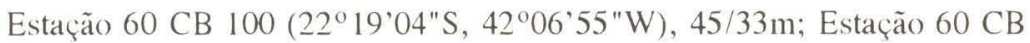
$101\left(22^{\circ} 58^{\circ} \mathrm{S}, 42^{\circ} 06^{`} \mathrm{~W}\right), 50 \mathrm{~m}$.

Lutjanidae

Lutjamus griseus ( Linnaeus, 1758) - Caranha

Estação $60 \mathrm{CB} 101$ (220 $\left.58^{\circ} \mathrm{S}, 42^{\circ} 06^{\circ} \mathrm{W}\right), 50 \mathrm{~m}$.

\section{Gerreidae}

Gerres aprion, Cuvier, 1829 - Carapicú

Estacão 60 CB 100 (22\%19’04"S, 42\%06 55"W ), 45/33m; Estação 60 CB $101\left(22^{\circ} 58^{\circ} \mathrm{S}, 42^{\circ} 06^{\circ} \mathrm{W}\right), 50 \mathrm{~m}$.

\section{Haemulidae}

Conodon nobilis ( Linnaeus, 1758) - Roncador

Estação 52 CP 89 (19³4’S, 39³2’W), 48/34m; Estação 60 CB 101 (22 $\left.{ }^{\circ} 58^{\prime} \mathrm{S}, 42^{\circ} 06^{\prime} \mathrm{W}\right), 50 \mathrm{~m}$.

Orthopristis ruber (Cuvier, 1830) - corcoroca-da-pedra

Estação 60 CB 101 (220 $\left.58^{\circ} \mathrm{S}, 42^{\circ} 06^{\prime} \mathrm{W}\right), 50 \mathrm{~m}$.

\section{Sparidae}

Pagrus pagrus (Linnaeus, 1758) - pargo-rosa

Estação 60 CB 101 (220 $\left.58^{\circ} \mathrm{S}, 42^{\circ} 06^{\prime} \mathrm{W}\right), 50 \mathrm{~m}$.

\section{Sciaenidae}

Ctenosciaena gracilicirrhus (Metzelaar, 1919) - cangauá

Estação 52 CP 89 (19³4'S, 39³2'W), 34/48m; Estação 52 CP 89

(19³3’s, 39³4’W), $34 \mathrm{~m}$.

Cynoscion leiarchus (Cuvier, 1830) - pescada-branca

Estação $60 \mathrm{CB} 101\left(22^{\circ} 58^{\circ} \mathrm{S}, 42^{\circ} 06^{\circ} \mathrm{W}\right), 50 \mathrm{~m}$.

Equetus lanceolarus (Linnaeus, 1758) - bacalhau

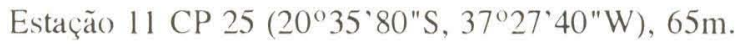

Isopisthus parvipinnis (Cuvier, 1830) - tortinha

Estação 5 I CP 88 (19\%35's, 3940’W), 20m.

Larimus breviceps (Cuvier, 1830) - boca-torta

Estação 51 CP 88 (19³5’s, 3940’W), 20m.

Macrodon ancylodon (Bloch \& Schneider, 1801) - pescada - foguete

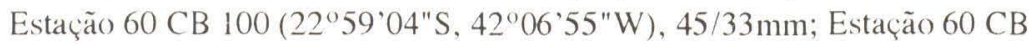
$101\left(22^{\circ} 58^{\circ} \mathrm{S}, 42^{\circ} 06^{\prime \prime} \mathrm{W}\right), 50 \mathrm{~m}$. 
Micropogonias furnieri (Desmarest, 1823) - corvina

Estação 51 CP 88 (19³5'S, 3940W), 20m; Estação 60 CB 101 (22 ${ }^{\circ} 58^{\circ}$ S, $\left.42^{\circ} 06^{\prime} \mathrm{W}\right), 50 \mathrm{~cm}$.

Nebris microps Cuvier, 1830 - pescada-banana

Estação 51 CP 88 (19³5'S, 3940’W), 20m.

Paralonchurus brasiliensis (Steindachner, 1875) - maria-luiza

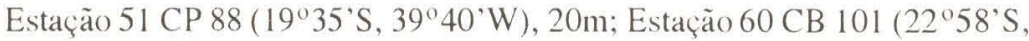
$\left.42^{\circ} 06^{\prime} \mathrm{W}\right), 50 \mathrm{~m}$.

Stellifer brasiliensis (Schultz, 1945) - cangoá

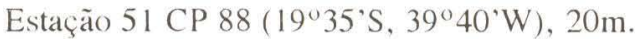

\section{Mullidae}

Pseudupeneus maculatus (Bloch, 1793) - salmonete

Estação 10 CP $23\left(20^{\circ} 42^{\circ} 00^{\prime \prime S}, 37^{\circ} 49^{\circ} 76^{\prime \prime W}\right)$, 52m; Estação 11 CP 25 $\left(25^{\circ} 35^{\prime} 80^{\prime \prime} \mathrm{S}, 37^{\circ} 27^{\prime} 40^{\prime \prime} \mathrm{W}\right), 65 \mathrm{~m}$.

\section{Chaetodontidae}

Chaetodon aculeatus (Poey, 1860) - peixe borboleta

Estação 10 CP $23\left(20^{\circ} 42^{\circ} 00^{\prime \prime S}, 37^{\circ} 49^{\prime} 76^{\prime \prime W}\right)$, 52m; Estação $11 \mathrm{CP}$ $\left(25^{\circ} 35^{\prime} 80^{\prime \prime} \mathrm{S}, 37^{\circ} 27^{\prime} 40^{\prime \prime} \mathrm{W}\right), 65 \mathrm{~m}$.

Chaetodon guyanensis Durand, 1860 - peixe-borboleta

Estação 11 CP $25\left(25^{\circ} 35^{\circ} 80^{\prime \prime} \mathrm{S}, 37^{\circ} 27^{\prime} 40^{\prime \prime} \mathrm{W}\right), 65 \mathrm{~m}$.

\section{Pomacanthidae}

Centropyge aurantonotus Burgess, 1974

Estação 14 DC 27 (20⒊'S 36॰42’W), 81 m; Estação 24 DC 42 (205' $\left.67^{\prime \prime} \mathrm{S}, 34^{\circ} 01^{\prime} 35^{\prime \prime} \mathrm{W}\right), 60 \mathrm{~m}$.

\section{Pomacentridae}

Chromis flavicauda (Günther, 1880)

Estação 31 CP 53 (20028'S, $28^{\circ} 51$ 'W), 69/54m; Estação 32 CP 53 $\left(20^{\circ} 28^{\prime} 53^{\prime \prime} \mathrm{S}, 28^{\circ} 51^{\prime} 65^{\prime \prime} \mathrm{W}\right), 120 \mathrm{~m}$.

\section{Labridae}

Xyrichtys novacula (Linnaeus, 1758)

Estação 31 CP 53 (2028'S, $28^{\circ} 5$ I 'W), 69/54m.

\section{Scaridae}

Cryptotomus roseus Cope, 1871

Estação 6 DC $15\left(21^{\circ} 37^{\circ} 75^{\prime \prime}\right.$ S, $\left.40^{\circ} 18^{\circ} 01^{\prime \prime W}\right), 37 \mathrm{~m}$. 


\section{Opistognathidae}

Opistognathus cuvieri Valenciennes, 1836

Estação 6 DC $15\left(21^{\circ} 37^{\prime} 75^{\prime \prime} \mathrm{S}, 40^{\circ} 18^{\circ} 01^{\prime \prime W}\right), 37 \mathrm{~m}$.

\section{Percophidae}

Bembrops heterurus (Ribeiro, 1903)

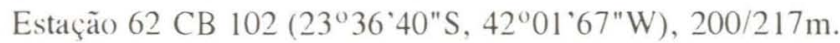

Percophis brasiliensis Quoy \& Gaimard, 1824 - tira-vira

Estação $60 \mathrm{CB} 100\left(22^{\circ} 59^{\circ} 04^{\prime \prime} \mathrm{S}, 42^{\circ} 06^{\circ} 55^{\prime \prime} \mathrm{W}\right), 45 / 33 \mathrm{~m}$; Estação $60 \mathrm{CB}$ 101 (22 $\left.58^{\circ} \mathrm{S}, 42^{\circ} 06^{\circ} \mathrm{W}\right) 50 \mathrm{~m} ; 1$ ex.: $242 \mathrm{~mm}$ CS, MNHN 1989-456.

\section{Mugiloididae}

Perseudopercis semifasciata (Cuvier, 1829) - namorado

Estação $61 \mathrm{CB} 102\left(23^{\circ} 07^{\prime} \mathrm{S}, 42^{\circ} 03^{\circ} \mathrm{W}\right), 100 \mathrm{~m}$.

\section{Clinidae}

Ribeiroclinus eigenmunni (Jordan, 1888)

Estação 30 DC 50 (22\% $\left.44^{\prime} \mathrm{S}, 31^{\circ} 49^{\circ} \mathrm{W}\right), 95 \mathrm{~m}$.

\section{Callionymidae}

Synchiropus agassizi (Goode \& Bean, 1888)

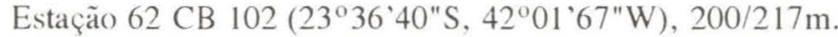

\section{Gobiidae}

Chriolepis vespa Hastings \& Boirtone, 1981

Estação 35 DC $59\left(20^{\circ} 30^{\prime} 32^{\prime \prime} \mathrm{S}, 21^{\circ} 18^{\prime} 58^{\prime \prime} \mathrm{W}\right), 61 \mathrm{~m}$.

Coryphopterus glaucofraenum Gill, 1863

Estação 10 DC 24 (20⒋ $\left.41^{\prime} 81^{\prime \prime S}, 37^{\circ} 49^{\prime} 55^{\prime \prime W}\right), 45 \mathrm{~m}$; Estação 17 DC 30 $\left(20^{\circ} 26^{\circ} 72^{\prime \prime S}, 36^{\circ} 17^{\prime} 30^{\prime \prime} \mathrm{W}\right), 60 \mathrm{~m}$; Estação 23 DC 41 (203' $50^{\prime \prime} \mathrm{S}, 34^{\circ} 42^{\prime}$

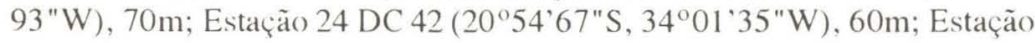

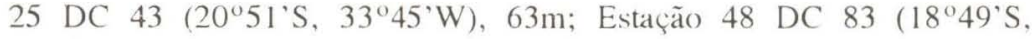
$\left.37^{\circ} 58^{\circ} \mathrm{W}\right), 60 \mathrm{~m}$.

Gobionellus smaragdus (Valenciennes, 1837)

Estação 30 DC 50 (204ㄴ $\left.\mathrm{S}, 31^{\circ} 49^{\prime} \mathrm{W}\right), 85 \mathrm{~m}$.

\section{Trichiuridae}

Trichiurus lepturus Linnaeus, 1758 - espada

Estação 60 CB $100\left(22^{\circ} 59^{\circ} 04^{\prime \prime} \mathrm{S}, 42^{\circ} 06^{\circ} 55^{\prime \prime} \mathrm{W}\right), 45 / 33 \mathrm{~m}$. 


\section{Stromateidae}

Prepilus paru (Linnaeus, 1758)

Estação 60 CB 100 (22 $\left.{ }^{\circ} 59^{\circ} 04^{\prime \prime} \mathrm{S}, 42^{\circ} 06^{\circ} 55^{\prime \prime W}\right), 45 / 33 \mathrm{~m}$.

\section{Bothidae}

Ancylopsetta cycloidea Tyler, 1959

Estação 52 CB 89 (19³3'S, 39³4'W), 34m; Estação 52 CB 90 (19³3'S, $\left.39^{\circ} 34^{\prime} \mathrm{W}\right), 34 \mathrm{~m}$; Estação $60 \mathrm{CB} 101$ (22 $\left.{ }^{\circ} 58^{\prime} \mathrm{S}, 42^{\circ} 06^{\prime} \mathrm{W}\right), 50 \mathrm{~m}$; Estação 61 CB $102\left(23^{\circ} 75^{\prime} \mathrm{S}, 42^{\circ} 03^{\circ} \mathrm{W}\right), 100 \mathrm{~m}$.

Bothus lunatus (Linnaeus, 1758)

Estação 31 CP 53 (2028'S, 28 51'W), 69/54m; Estação 32 CP 54 $\left(20^{\circ} 28^{\prime} 53^{\prime \prime} \mathrm{S}, 28^{\circ} 51^{\prime} 65^{\prime \prime} \mathrm{W}\right), 120 \mathrm{~m}$; Estação $52 \mathrm{CB} 89$ (19 ${ }^{\circ} 34^{\prime} \mathrm{S}, 39^{\circ}$ $\left.32^{\prime} \mathrm{W}\right), 34 / 48 \mathrm{~m}$; Estação 52 CB 90 (19³3'S, 39³4'W), 34m; Estação 60

CB $100\left(22^{\circ} 59^{\circ} 04^{\prime \prime} \mathrm{S}, 42^{\circ} 06^{\circ} 55^{\prime \prime} \mathrm{W}\right), 45 / 33 \mathrm{~m}$.

Bothus ocellatus (Agassiz, 18931)

Estação 32 CP 54 (2028’53"S, 28 5 I $\left.^{\circ} 65^{\prime \prime W}\right), 120 \mathrm{~m}$; Estação 52 CB 90, (19³3’s, 39³4’W), 34m.

Etropus crossotus Jordan \& Gilbert, 1881

Estação 60 CB 100 (22 $\left.59^{\prime} 04^{\prime \prime S}, 42^{\circ} 06^{\prime} 55^{\prime \prime W}\right), 45 / 33 \mathrm{~m}$.

Etropus longimamus Norman, 1933

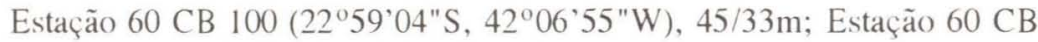
101 (22 $\left.{ }^{\circ} 58^{\prime} \mathrm{S}, 42^{\circ} 06^{\prime} \mathrm{W}\right), 50 \mathrm{~m}$; Estação 61 CB 102 (230.' $100 \mathrm{~m}$.

Hippoglossina bollmani Meek \& Hildebrand, 1928

Estação 60 CB 101 (22 $\left.58^{\circ} \mathrm{S}, 42^{\circ} 06^{\circ} \mathrm{W}\right), 50 \mathrm{~m}$.

Monolene antillarum Nomran, 1933

Estação 62 CB 103 (23ํำ $\left.40^{\prime \prime} \mathrm{S}, 42^{\circ} 01^{\circ} 67^{\prime \prime W}\right), 200 / 217 \mathrm{~m}$.

Paralichrys bicyclophorus Ribeiro, 1903

Estação 60 CB 100 (22ํำ'04"S, 4206'55"W), 43-33m; Estação 60101 (22ำ $\left.58^{\prime} \mathrm{S}, 42^{\circ} 06^{\circ} \mathrm{W}\right), 50 \mathrm{~m}$.

Paralychrys brasiliensis (Ranzani, 1840) - linguado

Estação 60 CB 101 (22ํำ'S, $\left.42^{\circ} 06^{\prime} \mathrm{W}\right), 50 \mathrm{~m}$.

Syacium micrurtm Ranzani, 1840 - Linguado-da-areia

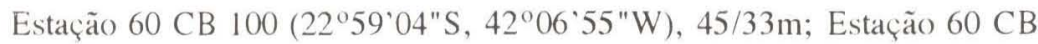

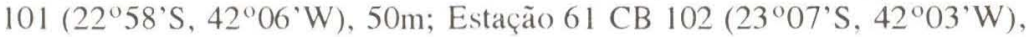
$100 \mathrm{~m}$.

Xystreurys rasile (Jordan, 1890)

Estação $61 \mathrm{CB} 102\left(23^{\circ} 07^{\prime} \mathrm{S}, 42^{\circ} 03^{\circ} \mathrm{W}\right), 100 \mathrm{~m}$; Estação 61 CB 102 $\left(23^{\circ} 07^{\prime} \mathrm{S} 42^{\circ} 03^{\prime} \mathrm{W}\right), 100 \mathrm{~m}$. 


\section{Cynoglossidae}

Symphurus ginshurgi Menezes \& Benvegnù, 1976

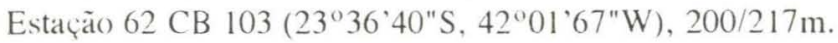

Symphurus plagusia (Bloch \& Schneider, 1801) - Lingua - de - mulata

Estação 13 DC 26 (2021'1 1"S e 3659’07"W), 97.5mm; Estação 61 CB $102\left(23^{\circ} 07^{\prime} \mathrm{S}, 42^{\circ} 03^{\prime} \mathrm{W}\right), 100 \mathrm{~m}$.

Symphurus trewavasae Chahanaud, 1948

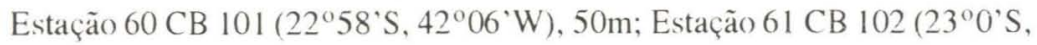
$\left.42^{\circ} 03^{\prime} \mathrm{W}\right), 100 \mathrm{~m}$.

\section{Soleidae}

Gymnachirus mudus Kaup, 1858

Estação $60 \mathrm{CB} 101\left(22^{\circ} 58^{\circ} \mathrm{S}, 42^{\circ} 06^{\prime} \mathrm{W}\right) 50 \mathrm{~m}$.

\section{Monacanthidae (Balistidae)}

Cantherhines pullus (Ranzani, 1842)

Estação 10 CP 23 (2042’00"S, 37²9’76"W), 52m.

Monacarthus ciliatus (Mitchill, 1818) - pirá-aca

Estação 48 CP 84 ( $\left.18^{\circ} 49^{\prime} \mathrm{S}, 37^{\circ} 58^{\prime} \mathrm{W}\right), 60 \mathrm{~m}$; Estação 51 CP 88 (19³5'S,

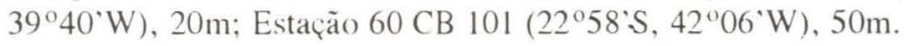

Stenophanolepis hispidus (Linnaeus, 1758) - Porquinho

Estação 10 CP $23\left(20^{\circ} 42^{\circ} 00^{\prime \prime S}, 37^{\circ} 49^{\circ} 76^{\prime \prime W}\right), 52 \mathrm{~m}$; Estação 60 CB 100

(22 $\left.{ }^{\circ} 59^{\circ} 04^{\prime \prime S}, 42^{\circ} 06^{\circ} 55^{\prime \prime W}\right), 45 / 33 \mathrm{~m}$; Estação $60 \mathrm{CB} 101\left(22^{\circ} 58^{\circ} \mathrm{S}, 42^{\circ}\right.$

$\left.06^{\circ} \mathrm{W}\right), 50 \mathrm{~m}$; Estação $61 \mathrm{CB} 102\left(23^{\circ} 07^{`} \mathrm{~S}, 42^{\circ} 03^{\prime} \mathrm{W}\right), 100 \mathrm{~m}$.

Xanthichthys ringens (Linnaeus, 1758)

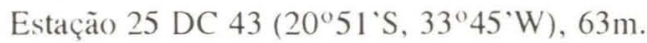

\section{Tetraodontidae}

Sphoeroides testudineus (Linnaus, 1758) - baiacú-mirim

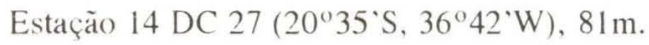

\section{Diodontidae}

Chilomycterus antllarum Jordan \& Rutter, 1897

Estação 60 CB 101 (22059`04"S, 42º6 55"W); 45/33m.

Chilomycterus spinosus Linnaeus, 1758 - baiacú de espinho

Estação $60 \mathrm{CB} 101$ (22 $\left.{ }^{\circ} 58^{\circ} \mathrm{S}, 42^{\circ} 06^{\circ} \mathrm{W}\right), 50 \mathrm{~m}$.

Diodon holocanthus Linnaeus, 1757 - baiacú de espinho

Estação 10 CP $23\left(20^{\circ} 42^{\prime} 00^{\prime \prime S}, 37^{\circ} 49^{\prime} 76^{\prime \prime W}\right)$, 52m; Estação 11 CP 25

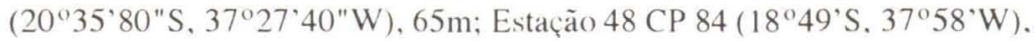
$60 \mathrm{~m}$. 


\section{CONSIDERAC̣ōES FINAIS}

A amostra coletada de 107 espécies é relativamente diversificada, embora não chegue a ser representativa da fauna ictiológica da plataforma continental brasileira quando comparada ao material obtido pela campanha do "Tôko-Maru" (1956-1957), que capturou 118 espécies de peixes ósseos e 25 cartilaginosos (NAKAmura et al. 1963), ou a do "Calypso" 1961-1962, que capturou 250 espécies de peixes teleósteos (Roux 1973) e 23 de Condrichthyes (Roux 1979). Isto provavelmente se deve ao fato dos tipos de instrumentos utilizados na Campanha MD-55 Brasil, como o arrastão "Chalut Blake", operaram apenas em fundos móveis e sobretudo vasosos, o que limitou a captura de peixes maus nadadores que vivem parcialmente enterrados nos sedimentos, como os representantes das famílias Bothidae, Batrachoididae, Cynoglossidae e Soleidae. Os tubarões, dourados, atuns e outros bons nadadores, não foram representados nas capturas realizadas na plataforma continental. Mesmo os nadadores medíocres, como as raias, conseguiram escapar do tipo de instrumento utilizado, considerando-se que apenas três exemplares jovens destas foram capturados. Em suma, o arrastão de vara "Chalut à perche" não se revelou satisfatório, sendo que uma abordagem mais ampla sobre os problemas relacionados ao equipamento de coleta utilizado encontra-se no relatório da Campanha (SÉRET \& ANDREATA 1988).

Entre 20-200/217 metros de profundidade, o material ictiológico coletado foi relativamente reduzido, tanto qualitativa como quantitativamente, o que provavelmente se deve aos inadequados instrumentos utilizados SÉRET \& ANDREATA (op. cit.). Nenhuma espécie nova de peixe foi observada, tendo sido registrado uma única ocorrência nova para o Atlântico Ocidental sul: Zenion hololepsis. Registrou-se, porém, ampliações dos limites de distribuição geográfica para Myrophis frio e Prionotus nudigula. O material coletado nas montanhas submarinas, Vitória/Trindade e Martin Vaz, ainda que relativamente pouco representativo, sugere serem as espécies idênticas às que ocorrem na plataforma continental brasileira.

AGRADECIMENTOS. Agradecemos aos professores doutores Jeanele Maron Ramos e Alain Guille. responsáveis pela campanha oceanográfica MD-55/ Brasil. por ter nos convidado a participar da mesma. Aos professores Mareo Antonio dos Santos. Maria Helena Carvalho da Silva Frederico Werneck Kurtz da Universidade Santa Úrsula pela ajuda prestada na coleta do material, a Jorge Alves de Oliveira do IBAMA. pelas informaçōes e empréstimo de hibliografia, ao Professor Dr. Gustavo W. Nunan do Museu Nacional do Rio de Janciro pela revisão do texto e sugestỏes e ao professor Philip C. Scott da Universidade Santa Úrsula pela versão do resumo em inglês.

\section{REFERÊNCIAS BIBLIOGRÁFICAS}

Alves, L.B. DE O.; M. Yesaki \& J.G. Gueran. 1973. Riobaldo - Cruzeiro R6/73. Rio de Janeiro, FAO/PNUD/SUDEPE, 5p.

Amaral, J.C. 1973. Riobaldo-Cruzeiro RIO/73. Rio de Janeiro, FAO, PNUD, SUDEPE, 6p. 
BEnVEGNÙ-LÉ, G. DE Q. 1978. Distribuição dos peixes teleósteos marinhos demersais na plataforma continental do Rio Grande do Sul. Tese de Doutorado, não publicada, Instituto de Biociências, Universidade de São Paulo, São Paulo, 94p.

BULlis JR. H.R. \& J.R. ThOMPSON. 1959. Shrimp exploration by the M/V Oregon along the northeast coast of South America. Commercial fisheries. Fish Wildlife Service 21 (11): 1-9.

Carvalho, V.A. DE \& E.J. Victer. 1974. Riobaldo; Relatório síntese; CNPq. Rio de Janeiro, FAO, PNUD, SUDEPE, 16p.

Chao, L.N.; L.E. Pereira; J.P. Vieira; M.A. Bemvenuti \& L.P.R. Cunha. 1982. Relação preliminar dos peixes estuarinos e marinhos da Lagoa dos Patos e região costeira adjacente, Rio Grande do Sul, Brasil. Atlântica, Rio Grande, 5 (1): 67-75.

Chao, L.N.; L.E. Pereira \& J.P. Vieira. 1987. Bioecology of fishes in the estuary and adjacent coastal areas of the Patos Lagoon, Brasil: a baseline study, p.429-450. In: A. YãneZ-ARANCIBIA (ed.). Fish comunity ecology in the estuaries and coastal lagoons - Towards an ecossistem integration. México, Univ. Nal. Autón. México, 646p.

Eskinazi, A.M. \& H. Lima. 1968. Peixes marinhos do Norte e Nordeste do Brasil, coletados pelo Akaroa, Canopus e NOc. Almirante Saldanha. Arq. Est. Biol. Mar. Univ. Fed. Ceará 8 (2): 163-172.

GüNTHER, A. 1889. Report on the pelagic-sea fishes collected by H. M.S. Challenger during the years 1873-1876. Challenger Report, (Zoology) 31: 1-47.

Guille, A. \& J.M. Ramos. 1988 - MD 55/Brésil à bord du "Marion Dufresne", 6 mai - 2 juin 1987. Terres Australes et Antarctiques Françaises (Mission de Recherche) et Universidade Santa Úrsula, Brasil. Les rapports des campagnes à la mer, 87-03: 1-198.

KARRER, C. 1968. Über Erstnachweise und seltene Arten von Fischen aus dem Südatlantik (argentinische - südbrasilianische Küste). 1 Mitteilung. Zool. Jh. Syst. Jena 95: 542-570.

Matsuura, Y. 1971. A study of the life history of brazilian sardine, Sardinella aurita, I. Distribution and abundance of sardine eggs in the region of Ilha Grande, Rio de Janeiro. Bolm. Inst. Oceanogr., São Paulo, 20 (1): 33-60.

1975. A study of the life history of brazilian sardine, Sardinella brasiliensis, III. Development of sardine larvae. Bolm. Inst. Oceanogr., São Paulo, 24: 17-29.

MCEACHRAN, J.D. 1983. Results of the research cruises of FRV "Walther Herwig" to South America. LXI. Revision of the South American skate genus Psamobatis Günther, 1870 (Elasmobranchii: Rajiformes Rajidae). Arch. Fisch. Wiss. 34 (1): 23-80.

Menezes, N.A. 1971. Relação dos peixes ósseos coletados durante os cruzeiros do navio oceanográfico "Prof. W. Besnard" costas do Rio Grande do Sul. Contribuições Avuls. Inst. Oceanograf. Univ. S. Paulo, série Ocean. Biol., 
(25): 44-60.

Nakamura, I.; T. Uemura; T. Yatsuca; S. Seki \& M. Murano. 1963. The investigation of the trawl fishing ground on the continental shelf off the Brazil. Tokyo, Fishery University, 388p.

. 1969. Relatório das pesquisas nas áreas de pesca do Brasil pelo navio oceano- gráfico Tôko-Maru. Belém, Ministério do Interior, SUDAM, 213p.

Nelson, J.S. 1984. Fishes of the world. New York, John Wiley, 2nd ed., 523p.

Nomura, H. \& J. Fausto-Filho. 1966. Shrimp Survey in Costal an off shore waters of Northeanastern and Northern Brasil. Arq. Est. Biol. Mar. Univ. Fed. Ceará, Fortaleza, 6 (1): 15-29.

OlenCO, P.G. DE. 1973. Descrição da prospecção ictiológica nas estações pesqueiras, p.85-20. In: XXXVIII Comissão oceanográfica NOc. Almirante Saldanha" (05/11 a 20/12/1966). Rio de Janeiro, Diretoria de Hidrografia e Navegação, Apêndice II.

OliveIRA, J.A. 1975. Ictiofauna marinha da região Norte-Brasil. Rio de Janeiro, Assessoria Divulgạą̃a SUDEPE, 25p.

. 1978. Relatório dos trabalhos técnicos efetuados durante a programação conjunta de pesquisa de Biologia e Pesca nas costas do Estado do Rio de Janeiro. Rio de Janeiro, SUDEPE, 6p.

1986. Informes ecológicos decorrentes da "Operação Cabo Frio IV"

Rio de Janeiro, Brasil. Rio de Janeiro, SUDEPE, 60p.

Ribeiro, A. DE M. 1903. Pescas do "Annie". A Lavoura, Bol. Soc. Nac. Agric. Rio de Janeiro 7: 150-197.

Ribeiro, P. DE M. 1961. Pescas do Tôko-Maru. Bol. Mus. Nac. Zoologia, Rio de Janeiro, 228: 1-18.

Roux, C. 1973. Campagne de la Calypso au large des côtes atlantiques de l’Amerique du Sud (1961-1962). Première partie (suite), 26. Poisson teléostéens du plateau continental brésilien. Ann. Inst. océanogr., Paris, 49 (supl.): 23-207.

1979. Campagne de la Calypso au large des côtes atlantiques de l'Amerique du Sud (1961-1962). Première partie (suite). 30. Poissons chondrichthyens du plateau continental brésiliens. Ann. Inst. océanogr., Paris, 55 (supl.): 111-130.

SADOWSKI, V. 1971. Relação dos peixes cartilaginosos capturados pelo navio

"Prof. W. Besnard" ao longo da costa do estado do Rio Grande do Sul (Programa Rio Grande do Sul). Contrções. Inst. Oceanogr. Univ. S. Paulo, ser. Ocean. Biol., 25: 40-43.

SÉret, B. \& J.V. Andreata. 1988. Les poissons, p. 151-158. In: A. GuIlle \& J.M. RAMOS (eds). Les rapports des campagnes à la mer n 87-03: MD-55 Brésil à bord du "Marion Dufresne"6 mai - 2 juin 1987, 198p.

Paris, 16 (1): 81-100.

Vazzoler, G. 1975. Distribuição da fauna de peixes demersais e ecologia dos Sciaenidae da plataforma continental brasileira, entre as latitudes $29^{\circ} 21^{\prime} \mathrm{S}$ 
(Torres e 3344'S (Chui). Bolm. Inst, Oceanogr. Univ. S. Paulo 24: 85-169. Vazzoler, A.E.A. de M.; F.M. de S. Braga; E. KawaKami; P.T.M.

Cunninghan; M.M. Bittencourt \& J.X. DE Mesquita. 1978. Cruz del sur - cruzeiro 1/78. Rio de Janeiro, FAO, PNUD, SUDEPE, 109p.

Recebido em 18.I.1995; acelto em 04.X.1995. 